Their masses can be estimated from the 'Weinberg angle' measured in neutrino interactions. At present predictions of the $\mathrm{Z}^{\circ}$ and the $\mathrm{W}^{+}$mass are $89 \pm 5 \mathrm{GeV}$ and $78 \pm 6 \mathrm{GeV}$, respectively. Single beams of about $45 \mathrm{GeV}$ should be sufficient to produce $\mathrm{Z}^{\circ} \mathrm{S}$ in association with other particles but the full machine energy will be required to create a $\mathrm{W}^{+} \mathrm{W}^{-}$pair.

For technical and cost reasons, the original CERN design study (LEP-70, completed this summer) was optimised for an energy of $70 \mathrm{GeV}$; but it is now being redone for $80-85 \mathrm{GeV}$ per beam. The size and cost of any such machine increase with its maximum energy, and LEP-70 already had a circumference of about $30 \mathrm{~km}$. However, valuable experience was gained from the LEP-70 design exercise. In particular the cost sensitivity of machine parameters and components can now be assessed more reliably.

Wolfgang Schnell, of the CERN ISR division, has already proposed some interesting ways of reducing the construction cost of the future accelerator. LEP will have more than 2,500 dipole bending magnets to keep the particles on their orbits. The bending power of each magnet is low because too high a track curvature would make the electrons lose too much of their energy by synchrotron radiation. Conventionally, magnets are made by precisely stacking hundreds of thin steel plates and welding them together. But the low magnetic fields required for LEP (to reduce bending, synchroton radiation, and running costs) can be obtained by bonding a smaller number of steel plates in concrete, thereby dispensing with a lot of steel and fabrication cost.

Further, the particle beams have to be refocused repeatedly to counteract the natural tendency of beams to diverge. More than 800 quadrupole magnets are foreseen for this task. A similar number of higher multipole magnets completes the set of tools which allow machine operators to tune the beams and to overcome instabilities. Using anodized aluminium in the manufacture of quadrupole coils, in place of copper, would save much cost and production effort; detailed investigations into the properties of such coils are already under way.

The costliest item of the machine is the radio-frequency system which will accelerate the electron beams. Its components, klystrons and accelerating cavities, stretch over $1.5 \mathrm{~km}$ and their total power consumption at $70 \mathrm{GeV}$ will be over $70 \mathrm{MW}$. About $19 \mathrm{MW}$ of this will simply be lost in synchrotron radiation, a feature which is negligible in all existing proton accelerators. When a bunch of electrons passes through the cavities, it picks up energy from the applied RF power and thus is accelerated. But the four bunches in each beam spend most of their time outside the accelerating cavities, and the applied power is lost. An ingeneous method to reduce these losses is to couple a low-loss mode storage cavity to a set of accelerating cavities. Energy can then be saved by constantly transferring power between the accelerating and storage cavities.

By itself, this scheme would not help on component cost but it would either reduce running cost, or alternatively allow a higher maximum beam energy. superconducting RF cavities is curto become available in a few years' time; they will eventually allow the beam energy to be raised to about $130 \mathrm{GeV}$. The upper limit is set by the synchrotron radiation power that can be absorbed by the vacuum chamber, and by the field gradient ilmitations in the accelerating cavities. Research into super conducting RF cavities is currently carried out under a joint CERNDESY programme, and a first test cavity is to be installed into DORIS by the end of 1979. However, it is unlikely that this technology will be sufficiently mastered for mass production for the first phase of LEP.

Great importance is also attached to the planning of experimental installations and facilities. Experience at DORIS and SPEAR has shown that the cost of experimental detectors and associated electronics and computers amounts to a sizeable fraction of the total machine cost. Furthermore, the average number of charged particles produced in an electron-positron annihilation at these energies is of the order of 20. Very complex detectors are required to track that many particles, and to identify them unambiguously. So a major effort must be made to reduce the cost of such devices.

Konrad Guettler

\section{Fermilab looks to the future}

\section{David Dickson visits the Fermi National Laboratory and talks to its new director, Dr Leon Lederman (right)}

THE past year has not been an easy one for the Fermi National Laboratory )Fermilab) at Batavia, just outside Chicago. Twelve months ago Fermilab's then director, Dr Robert R. Wilson, was pushing hard for the rapid completion of the next phase in Fermilab's development. This is to be the construction of a second ring of superconducting magnets which, with their increased field, will allow energies of up to $1,000 \mathrm{GeV}$ to be reached, twice existing energies.

Last November, frustrated by indications that the Department of Energy would not provide him with sufficient funds for the rapid construction of the Doubler, Dr Wilson threatened to resign if such funds were not included in Fermilab's budget for the fiscal year 1979. But the department stood firm, providing Wilson with only $\$ 15$ million of the $\$ 30$ million that he had requested. And in February he resigned as threatened, citing the department's "indecisive and "subminimal" support for the Doubler project.

The whole affair left the US high energy physics community feeling somewhat uncomfortable. Many of those who had admired Dr Wilson's achievements in establishing Fermilab were uncertain about the strategy that he had chosen to adopt. Dr Wilson also left a legacy at the laboratory where many felt experimental programmes had been unfairly squeezed to allow development work on the Doubler to proceed as rapidly as possible.

The task of putting all the pieces back together again now rests with Fermilab's new director, Dr Leon Lederman, whose appointment was announced in October and who takes over the position full-time next 1 June (although already working several days a week at the laboratory).

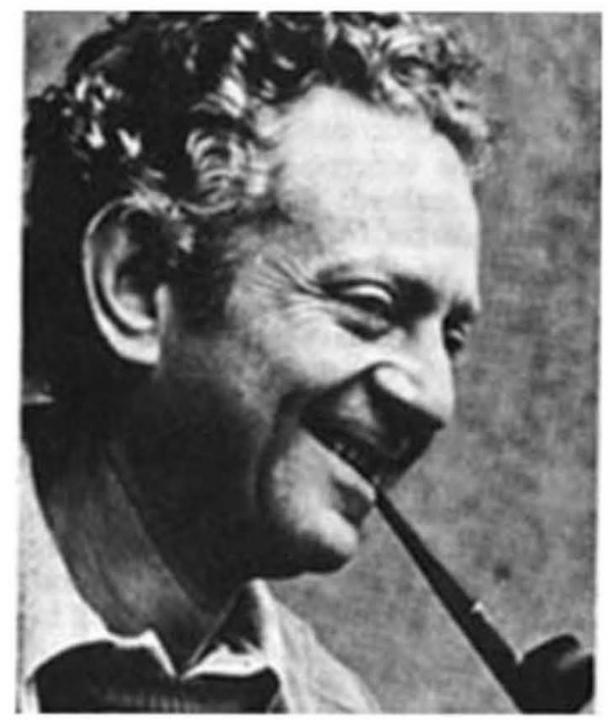

of physics at Columbia University and director of the university's Nevis accelerator. $\mathrm{He}$ has also been closely involved with Fermilab since its inception; he was a member of the team which selected the site from over a hundred possibilities in the 1960s, and he also led the investigators who discovered the upsilon particle in the summer of 1977. 
Widely known nat only as a successful experimentalist but also the owner of a quick wit-asked where the laboratory is going, his immediate response is that "It's going to stay in Illinois-I hope"--Dr Lederman has few illusions about the problems that lie ahead.

At the heart of many of these lies the issue of funding. Tight budgets have squeezed high energy physics as hard as other fields of science. Dr Lederman says that "basic research is being treated rather well by this administration"; but he points out that with three large accelerator projects now under way, the whole US high energy physics programme is "pushing very hard against its ceiling", and that "if the budget was even $5 \%$ higher, it would be an enormous relief".

\section{High risks}

The Energy Doubler will require a ring of 1,000 superconducting magnets, each 7 metres long. Although recognising that, with so little known about such technology, the field was one of high risks, Dr Wilson felt that the design of the magnets could be refined as the first ones were being produced (rather than waiting for a complete design before setting up production facilities).

However the gamble did not quite come off. Technical problems proved to be slightly greater than initially predicated-such as a slight movement iil some of the wires of the coils, affecting their superconducting properties-and took longer than anticipated to perfect.

As Dr Lederman puts it: "There were some concepts in economy that were not realised. We had thought that a much larger fraction of the original magnets would be adequate and that we would be able to instal those to make up one sixth of the ring. But not enough of them were of good quality; and that created a problem with funding".

Dr Lederman places stress on the need to maintain a high level of performance, not only with the Doubler but also with the existing accelerator. "We have at present a responsibility to provide a large fraction of the US high energy research community with 400 $\mathrm{GeV}$ protons, I have found attention to the programme has slipped somewhat and people have been travelling here from the East coast and the West coast and waiting for a machine that was broken down a bit too often."

With development work on the Doubler's magnets now completed, a team at Fermilab is now preparing a final detailed design of the accelerator, which is expected to be ready in January.
Until a few years ago, many of Fermilab's problems might have been solved by a quick visit to sympathetic Congressman, with a few million dollars subsequently added to budget appropriations. But this style of approach is no longer appropriate, particularly as the decision making role has shifted from the old Congressional Joint Atomic Energy Committeewhich produced much of the political support for early accelerators-to the Department of Energy's High Energy Physics Advisory Panel (HEPAP).

Earlier this year Dr John Deutch, director of the department's office of Energy Research, announced that the Office of Management and Budget had accepted a proposal to provide high energy physics with a consiant budget over the next few years. Within this limit, the department has left it largely up to HEPAP to advice on how the resources should be allocated; the result is that any major development requires the active backing of HEPAP if it is to get support in the administration.

"One of our current very high-level efforts is therefore to bring the physics community into a level of acceptance, understanding and support of the Doubler project", says Dr Philip Livdahl, head of the project and currently acting-director of Fermilab.

Dr Lederman has already asked for an outside, independent review of the whole Doubler project, and has invited scientists both at Fermilab and in the universities to present ideas for the type of experiments that they would like to carry out when the $1,000 \mathrm{GeV}$ level become available with the completion of the new ring.

A number of such ideas were discussed at a meeting of HEPAP which was held at Fermilab at the beginning of last week. It is said that the exercise had a beneficial effect on some of those who still have doubts about the Doubler project. The advisory panel has now asked the laboratory to prepare a detailed report on the ways in which the Doubler construction programme might be integrated with other activities, setting out the option at different budget levels so that it can assess the requests for greater support.

\section{Colliding with Japan}

From Fermilab's perspective, HEPAP's support is likely to be particularly important next year, since it is hoped that funds to begin construction of a colliding beam facility-involving collisions between particles in the existing ring and those circulating in the superconducting ring-can be included in the Department of Energy's budget request for 1981 .

One bright spot on the funding horizon is the proposed collaboration with Japanese scientists. High energy physics figures prominently in a programme of research collaboration in the energy sciences between the US and Japan that could be worth $\$ 1,000$ million over the next ten years.

Collaboration in high energy physics will take place at three different levels: carrying out joint experiments on existing facilities, joint research and development in accelerator design and technology, and-potentially the most ambitious point-funding of new facilities.

In the first category, Dr Lederman hopes the Japanese contribution will add those few percentage points to the research budget which would have a magnified effect on scientific productivity. As to the third, Japanese funds could help bring forward some of Fermilab's more long-term plans: in particular, the development of facilities for the production of highenergy anti-protons which could be injected into the same ring as the protons, but circulating in the opposite direction to give collision energies of 2,000 GeV.

\section{World Machine}

And there is an interest in high energy physics not only in Japan, but also in the Republic of China, which has recently sent a number of scientists to study at Fermilab. Dr Lederman has been closely involved in the activities of the International Committee for Future Accelerators, and in particular its scheme for the possible construction of a "world accelerator" in the 20,000 $\mathrm{GeV}$ range $(20 \mathrm{TeV})$, well beyond the economic scope of any one country.

"When we started there were only three areas of the world interested in the possibility of such a project: the US, the USSR and Western Europe. With three there are problem of cooperation; but with five the situation is much better, since it does not matter too much if one decides to drop out."

Dr Lederman is also understandably enthusiastic about the particular possibilities raised by the Japanese connection, and is a member of the Department of Energy working party which has been discussing details of the proposed collaboration between the two countries with Japanese scientists.

He feels that high energy physics could play an important role in Japanese society. "Talking to Japanese politicians and ministers, they seem to feel the lack of intellectual heroes, the role played by Einstein, Fermi, Newton and so on in the West. They do not have that heritage to inspire the young people." High energy physics, Lederman belives, might provide the inspiration. 